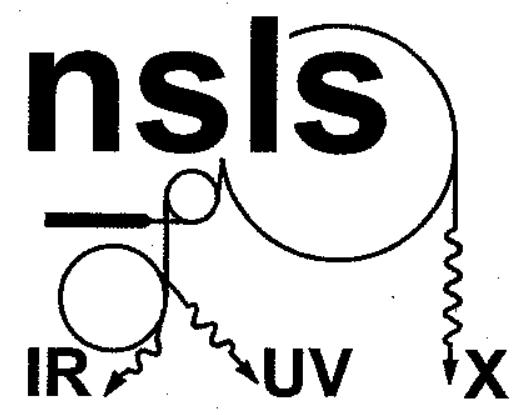

BNL Informal Report

BNL 71305-2004-IR

Comparison of Two Different Wavelength Tunging Schemes

In $A$

Seeded High-Gain FEL

T. Shaftan and L.H. Yu

National Synchrotron Light Source

Brookhaven National Laboratory

Upton, New York 11973

June, 2004

National Synchrotron Light SOURCe 


\section{DISCLAIMER}

This report was prepared as an account of work sponsored by an agency of the United States Government. Neither the United States Government nor any agency thereof, nor any of their employees, nor any of their contractors, subcontractors, or their employees, makes any warranty, express or implied, or assumes any legal liability or responsibility for the accuracy, completeness, or usefulness of any information, apparatus, product, or process disclosed, or represents that its use would not infringe privately owned rights. Reference herein to any specific commercial product, process, or service by trade name, trademark, manufacturer, or otherwise, does not necessarily constitute or imply its endorsement, recommendation, or favoring by the United States Government or any agency, contractor or subcontractor thereof. The views and opinions of authors expressed herein do not necessarily state or reflect those of the United States Government or any agency, contractor or subcontractor thereof. 


\title{
Comparison of Two Different Wavelength Tuning Schemes in a Seeded High-gain FEL
}

\author{
T. Shaftan and L.H. Yu \\ NSLS, BNL,Upton, NY 11973
}

Abstract

In the following text we analyze and compare results of the two publications ([1], [2]), dedicated to development of the tunable high-gain Free Electron laser (FEL), seeded by an external source. As a conclusion we summarize similarities and differences between these concepts.

This note is initiated by the polemics on the similarity of two different schemes of the wavelength tuning in a seeded high-gain FEL. Below we will be calling the scheme, presented by S. Biedron, S, Milton and H. Freund in Nuclear Instruments and Methods of 2001 ([1]), as the first $\left(1^{\text {st }}\right)$ scheme. The scheme that we developed and presented in the BNL preprint ([2]) will be called as the second $\left(2^{\text {nd }}\right)$ scheme.

The following analysis provides important information on similarities and differences between both techniques. We start by considering the first approach. As we understand, the goal of the Modular Approach is to make X-ray FEL design more flexible [1]. Usual linac-based FELs begin from a long linac with bunch compressor(s) followed by an FEL magnetic system. The essence of the Modular Approach is to break a machine into modules and then recombine these modules in a more efficient way (from the point of view of monetary and/or time constraints). The chapter 3.5 of [1] presents some basic example of Modular Approach.

We have studied the scheme, discussed in [1] and illustrated in Fig. 2. Even though very few details of the scheme were presented, it was still sufficient to obtain a complete qualitative picture. Let us begin with the usual phase space of the microbunched beam, which enters a radiator in a prebunched FEL (Fig. 1). The sharp spike (in blue) represents the longitudinal density bunching, which will be the main subject of interest in this discussion. The key principle of a seeded high-gain FEL optimization is to establish this kind of the electron beam phase space at the entrance of the radiator.

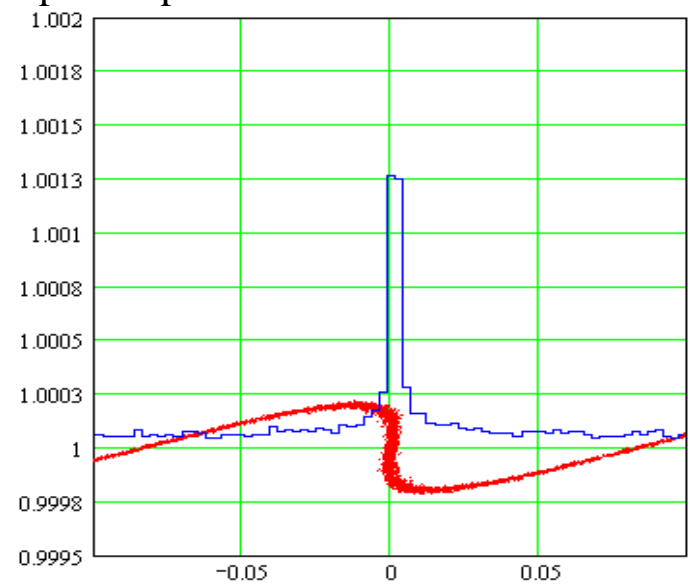

Fig. 1: Phase space of the bunched electron, suitable for FEL. In the following the scale for the bunching is 10 times finer as compared with Fig. 1 
The $1^{\text {st }}$ scheme is shown in Fig. 2 (Fig. 2 in NIM A) below. As can be readily determined from this picture, the beamline consists of the $1^{\text {st }}$ undulator, the chicane, the $1^{\text {st }}$ accelerator, the $2^{\text {nd }}$ chicane, the $2^{\text {nd }}$ accelerator and the $2^{\text {nd }}$ undulator. Now we will follow the scheme and find out what is the resulting phase space at the entrance to the $2^{\text {nd }}$ undulator. Figure 3 shows the phase space right after the first undulator.

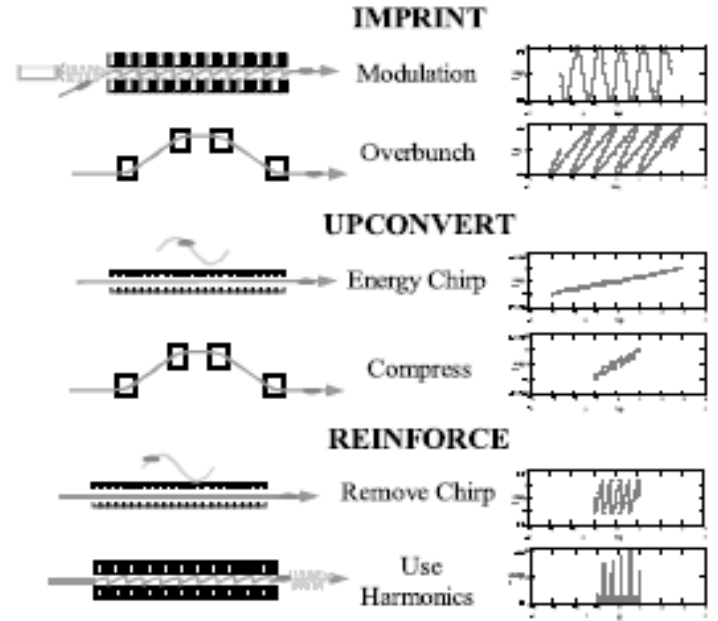

Fig. 2. Example $\mathrm{V}$-wavelength shifting concept.

Fig. 2: Wavelength shifting concept [1].

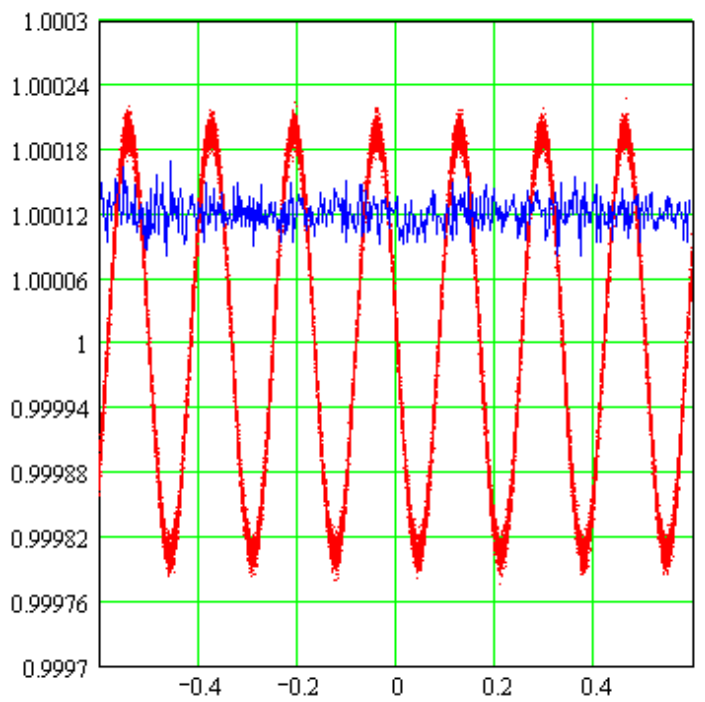

Fig. 3: Phase space after the first undulator.

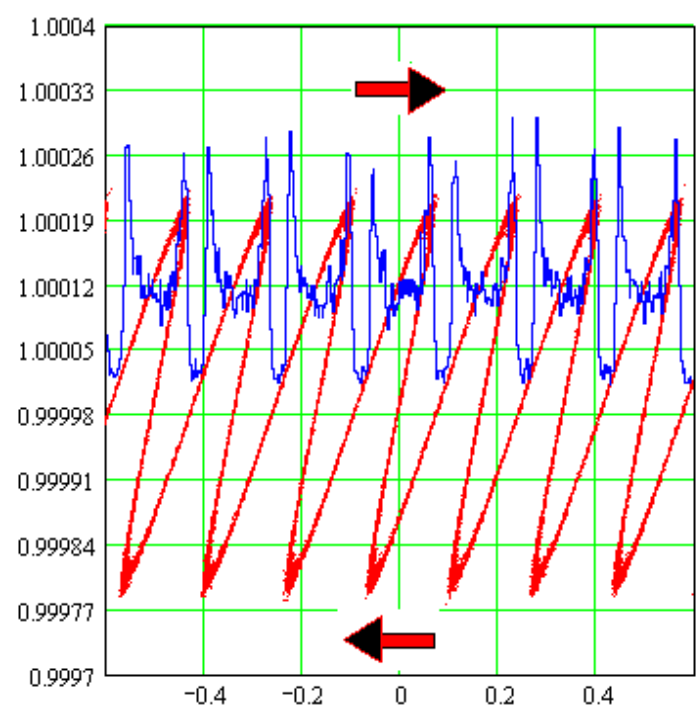

Fig. 4: Phase space after the first chicane.

As shown by the arrows in Fig. 4, as the electron beam moves through the chicane the particles with higher energy move forward along the bunch, and those with lower energy moves backwards $[3,4,5]$. Since a large amount of dispersion is used, the phase space is "overrotated" (as shown in Fig. 2 and in Fig. 4 above). 
Now the electron beam energy is increased in the $1^{\text {st }}$ accelerator, which is phased "slightly off-crest" [1]. Figures 5 and 6 show phase space and density profile of the chirped bunch for positive and negative chirps respectively.

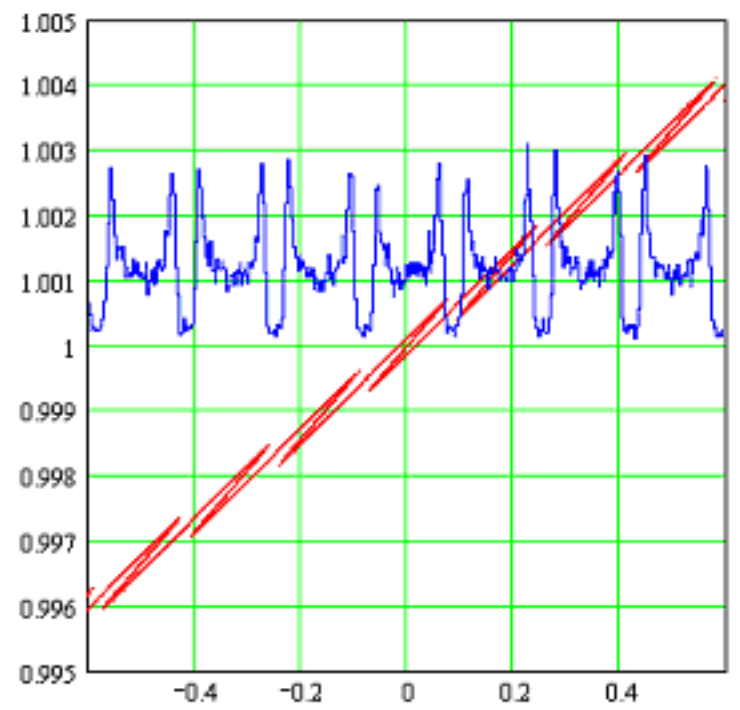

Fig. 5: Phase space after the first accelerator (positive dhimp). Blue durve shows the bunching content.

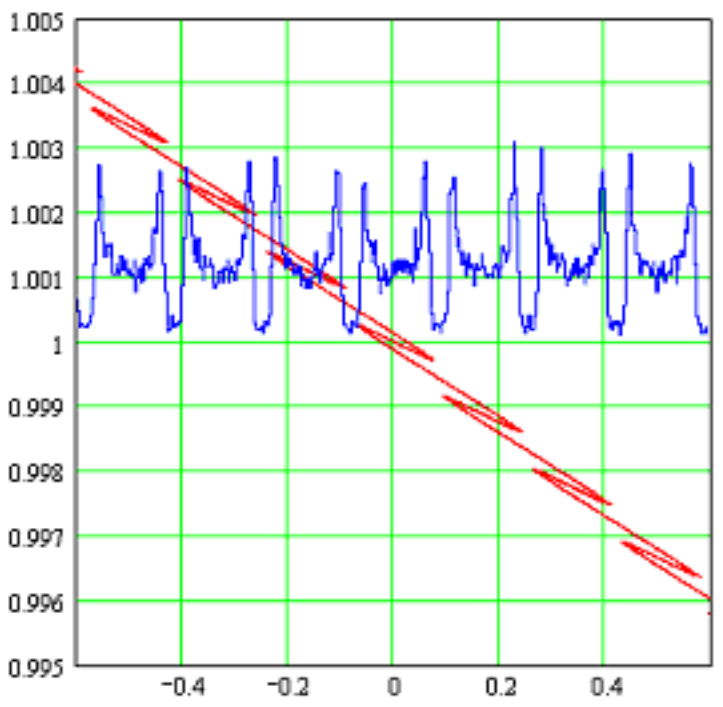

Fig. 6: Phase space after the first accelerator (negative chirp)

Let us now focus on the properties of a single slice of the electron beam, one radiation period in length. In Figs. 7 and 8, we show the phase space of a single slice under the same conditions as Figs. 5 and 6 (red) and the phase space in the second chicane $e^{1}$ (blue). The question is whether the $2^{\text {nd }}$ chicane will provide the desired density bunching as shown in Fig. 1.

\footnotetext{
${ }^{1}$ We note that, according to Fig. 2 of NIM A article [1], quite a strong chicane is needed and expected compression ratio in this scheme must be large.
} 


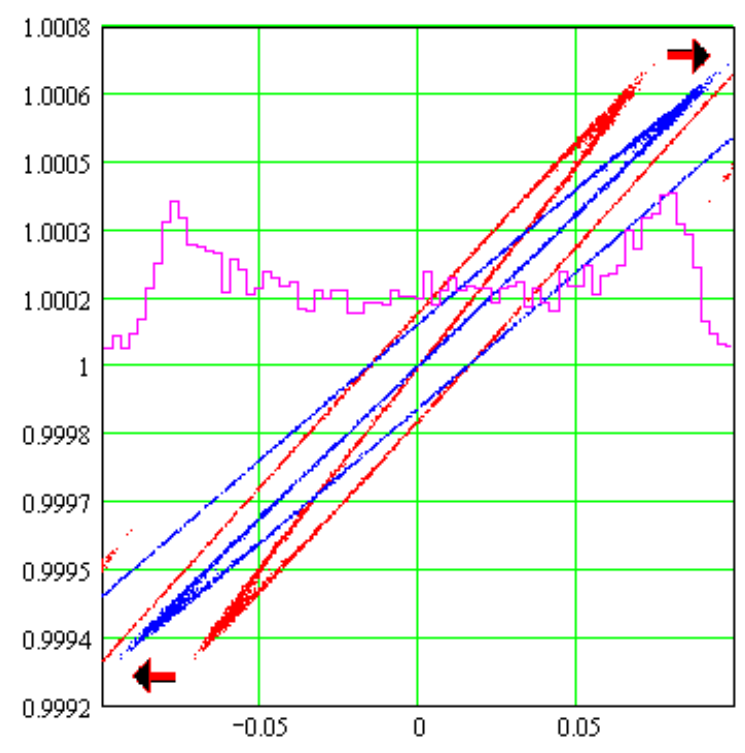

Fig. 7: Phase space of a single modulation period before and after the second chicane (positive chirp). Magenta curve shows the bunching content

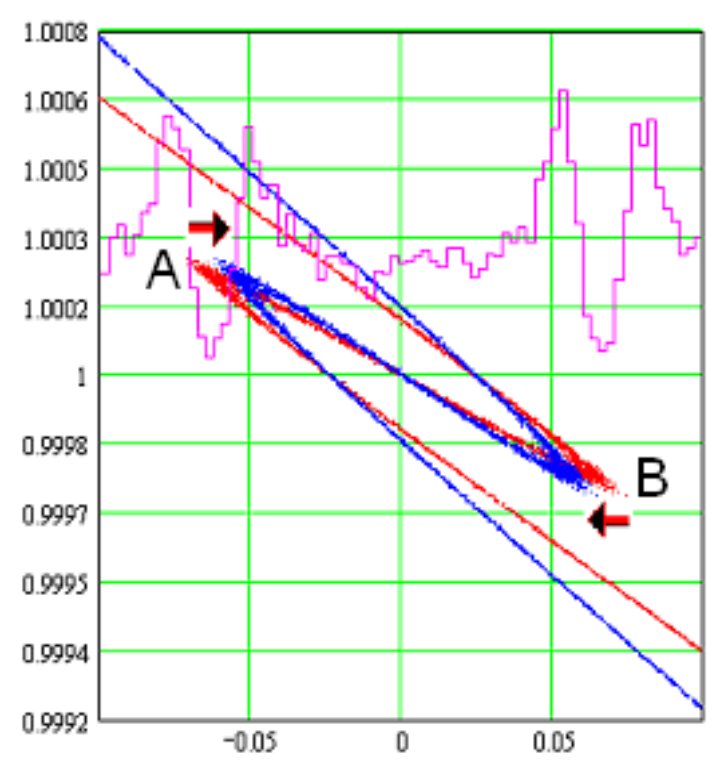

Fig. 8: Phase space of a single modulation period before and after the second chicane (negative chirp)

For the positive chirp (Fig .7), we observe the inability of the second chicane to create the desired microbunching as illustrated in Fig. 1. This is because in the chicane, particles with higher energy continue to move towards the head of the bunch, while the lower energy ones move to the rear. Hence the microbunching density is further reduced. As a result, while the beam is decompressed no useful microbunching content is created (compare with Fig. 1). We note that, while discussing this case in Fig. 2 in [1], a phase space was sketched with the desired bunching. This seems to us to be incorrect.

Now we examine in Fig. 8, the other sign of the chirp (which is not discussed in [1]). There is only one way to provide any bunching in the following $2^{\text {nd }}$ chicane: particle A must move up in energy higher than particle B (Fig. 8). It is easy to calculate the required chirp: $\mathbf{h}>\mathbf{4} \Delta_{\gamma} / \boldsymbol{\lambda}$, where $\Delta_{\gamma}$ is energy modulation amplitude and $\lambda$ is the modulation wavelength. Substituting some practical numbers $\left(\Delta_{\gamma}=0.1 \%, \lambda=400 \mathrm{~nm}\right)$ we get the value of the required chirp to be larger than $10000 \mathrm{~m}^{-1}$. Of course, it is unrealistic to provide this amount of chirp (most of it, apparently, must be removed after the second chicane). Acceleration between $1^{\text {st }}$ and $2^{\text {nd }}$ chicane may somewhat help with this, but energy gain of a large factor is required to solve the problem.

In the $2^{\text {nd }}$ chicane, again, particles with higher energy continue to move towards the head of the bunch, and the lower energy ones to the rear. It follows from Fig. 8, that this situation also doesn't create the desired bunching. In order to create the proper bunching the point A must be brought right above point $\mathrm{B}$. Before this can happen, the overall bunch will be compressed down to a smallest possible length. As easy imagine, at this moment the whole bunch will be broken into two gigantic spikes with extremely large peak current. During this compression-decompression collective effects will blow up the spikes, smashing any useful modulation or microbunching. Thus, this scenario does not work either. 
As a result, we may conclude that the Modular Approach does not provide necessary bunching content and, therefore, cannot be used for the desired goal.

Besides, even if it would be possible to overcome the principal difficulties, this FEL would never operate due to the next order limitations. We list some of them in the following paragraph.

1) Dramatic perturbations of the beam longitudinal phase space take place in two (!) bunch compressors. Any coherent and static effects (CSR, space charge, etc.) must ruin useful microbunching and destroy the beam emittance and energy spread [3].

2) Acceleration provided in the $1^{\text {st }}$ scheme may dramatically affect the beam quality.

3) Debunching due to transverse effects must be a killer in the complicated transfer line between undulators.

4) Compression ratio and the beam energy must be kept with a high accuracy to stay in resonance in the second undulator. We can only guess how unstable would be the FEL operation.

Now we discuss the second idea (concept) [2]. In the BNL preprint we begin from a general discussion about chirped beam FEL schemes, such as, SASE pulse compression and Chirped Pulse Amplification. Next we analyze the case of the chirped bunch, propagating through a single HGHG cascade (that is, through the standard optical klystron configuration) of HGHG FEL. It is essential that our scheme has nothing to do with Modular Approach. Oppositely, we always use one of the "basic modules" of Modular Approach: a single HGHG module (for the definitions see Chapter 2 of [1]).

We notice a compression of the bunch in the FEL system, due to its natural dispersion. According to the principle of optical klystron, the main amount of dispersion is localized in the dispersive section. In the following we maximize the dispersion section strength, define the FEL tuning range and discuss possible limitations of this scheme.

The main limitation of $2^{\text {nd }}$ scheme comes from the widening of the output FEL bandwidth due to the remaining energy chirp. Therefore we propose to include an auxiliary harmonic RF system into a single HGHG cascade. This RF system provides only chirp and only locally, which helps to overcome most of the limitations.

In the conclusion we propose to alter the output HGHG wavelength by this method, in combination with the standard method of changing the seed laser wavelength. We also mention the effectiveness of the $2^{\text {nd }}$ approach, since the HGHG FEL may generate radiation at the different harmonics of the seed laser wavelength. Thus, as a main conclusion, the HGHG FEL can be made completely tunable.

Comparing our idea (concept) with the Modular Approach, we observe:

1) $2^{\text {nd }}$ concept is based on a single HGHG cascade

2) $2^{\text {nd }}$ concept uses a very mild compression, since it employs the HGHG dispersive section, not chicanes

3) No acceleration in $2^{\text {nd }}$ concept

4) No "overrotation" in $2^{\text {nd }}$ concept

5) $2^{\text {nd }}$ concept: modest but symmetric tuning range

6) $2^{\text {nd }}$ concept is well estimated and proven to work 
7) $2^{\text {nd }}$ concept is tested experimentally; measured quantities are in a good agreement with estimates.

As it seems, the basic idea of the concepts is the same: compression of the modulated beam can be used to alter the output FEL wavelength. But, based on this comparison, we may conclude that the $2^{\text {nd }}$ concept is entirely different from the $1^{\text {st }}$ one.

\section{References}

1. S.G. Biedron, S.V. Milton, and H.P. Freund, "Modular Approach to Achieving the Next Generation Light Source,” Nuclear Instruments and Methods in Physics Research A 475 (2001) 401.

2. T. Shaftan and L.H. Yu, BNL preprint, BNL 720-34-2004-JA, Feb. 2004

3. M. Borland, Phys. Rev. ST Accel. Beams 4, 074201 (2001), Design and performance simulations of the bunch compressor for the Advanced Photon Source Low-Energy Undulator Test Line free-electron laser

4. M. Borland, J. Lewellen, S. Milton, LINAC2000, A Highly Flexible Bunch Compressor for the APS LEUTL FEL

5. M. Borland, talk, presented at ANL, June 3, 2002, Design Considerations for Linac FEL drivers 